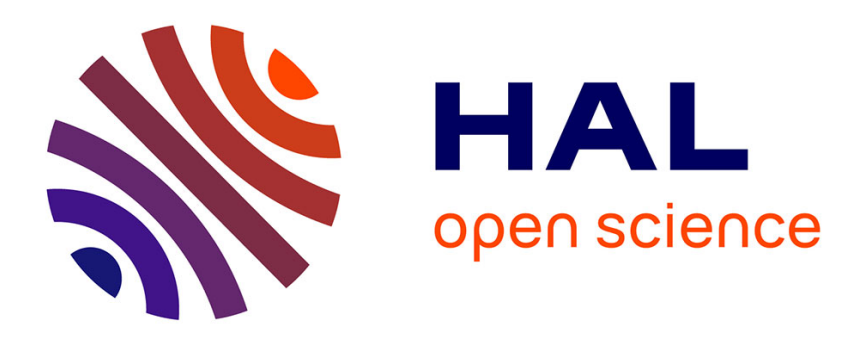

\title{
Toucher les dieux : rituels, expérience sensible et modes de contact avec le divin dans le monde grec
}

\author{
Adeline Grand-Clément
}

\section{To cite this version:}

Adeline Grand-Clément. Toucher les dieux: rituels, expérience sensible et modes de contact avec le divin dans le monde grec. Gaïa - Revue interdisciplinaire sur la Grèce archaïque, 2017, 20 (1), pp.199-222. 10.3406/gaia.2017.1732 . hal-02177978

\section{HAL Id: hal-02177978 \\ https://hal.science/hal-02177978}

Submitted on 9 Jul 2019

HAL is a multi-disciplinary open access archive for the deposit and dissemination of scientific research documents, whether they are published or not. The documents may come from teaching and research institutions in France or abroad, or from public or private research centers.
L'archive ouverte pluridisciplinaire HAL, est destinée au dépôt et à la diffusion de documents scientifiques de niveau recherche, publiés ou non, émanant des établissements d'enseignement et de recherche français ou étrangers, des laboratoires publics ou privés. 


\section{Toucher les dieux : rituels, expérience sensible et modes de} contact avec le divin dans le monde grec

Adeline Grand-Clément

\section{Citer ce document / Cite this document :}

Grand-Clément Adeline. Toucher les dieux : rituels, expérience sensible et modes de contact avec le divin dans le monde grec. In: Gaia : revue interdisciplinaire sur la Grèce Archaïque, numéro 20, 2017. Toucher le corps dans l'Antiquité. pp. 199-222;

doi : https://doi.org/10.3406/gaia.2017.1732

https://www.persee.fr/doc/gaia_1287-3349_2017_num_20_1_1732

Fichier pdf généré le 17/01/2019 


\title{
Résumé
}

L'anthropomorphisme des dieux grecs qui prévaut dans la tradition littéraire fondée par Homère et Hésiode rend possible une proximité physique, voire une forme d'intimité entre hommes et dieux, dans les récits qui les mettent en scène. Mais qu'en est-il des pratiques rituelles grecques effectives, qui ont précisément pour objectif d'établir le contact entre le monde terrestre et la sphère divine ? Le fidèle peut-il «toucher » le corps des dieux ? Et, mieux, cherche-t-il à le faire ? Telles sont les questions qui servent de départ à la réflexion. L'analyse porte ainsi sur les différents types de contacts physiques possibles entre les Grecs et les statues de leurs dieux, afin de déterminer dans quelle mesure ils permettent aux fidèles de «toucher » les dieux eux-mêmes. Les exemples étudiés, à partir de la documentation littéraire, épigraphique et archéologique, concernent principalement les époques archaïque, classique et hellénistique.

\begin{abstract}
Touching the Gods : Rituals, Sensory Experience and 'Physical' Interactions with the Divine in the Ancient Greek World.

The anthropomorphism of the Greek gods which prevails in the literary tradition founded by Homer and Hesiod, allows a physical proximity, even an intimacy, between mortals and Immortals, as can be read in the myths. But what about the ritual practices, which precisely aim at establishing a contact between the mortals and the divine world ? Can the devotee succeed in "touching" the gods ? And does he really intent to "touch" the god? These questions are the starting point of the paper. The analysis aims at sheding light on the different types of contacts that did exist between the Greeks and the statues of theirs gods, in order to determine to which extend these physical contacts were perceived as a means of touching the gods themselves. Most of the examples selected are taken from the literary, epigraphical and archaeological documentation ranging from the archaic to the roman period.
\end{abstract}




\title{
Toucher les dieux : rituels, expérience sensible et modes de contact avec le divin dans le monde grec
}

\author{
ADELINE GRAND-CLÉMENT \\ Université Toulouse 2 -Jean Jaurès
}

Face à la primauté dont bénéficie la vue, au sein de la tradition occidentale, historiens et anthropologues soulignent aujourd'hui la nécessité de repenser la place des autres registres du sensible pour mieux appréhender les systèmes de représentation et d'appréciation des différentes sociétés qu'ils étudient ${ }^{1}$. Les hellénistes contribuent à un tel renouvellement de la recherche, en s'intéressant notamment à l'univers olfactif et au paysage sonore des mondes anciens. Plus rares, en revanche, demeurent les recherches consacrées au toucher, si l'on excepte peut-être ce qui a trait au domaine médical ${ }^{2}$. Pourtant, la réhabilitation de la dimension haptique paraît nécessaire, surtout si l'on considère la forte valorisation dont bénéficie le corps au sein de la culture grecque ${ }^{3}$. L'absence de travaux d'envergure sur celui qu'Aristote considère dans son traité De l'âme comme le dernier sens, le sens le plus animal $^{4}$, s'explique sans doute par son caractère insaisissable ${ }^{5}$. En effet, le toucher, à la différence des autres domaines de la perception sensorielle, ne

1. Pour une anthropologie historique du sens du toucher, voir Classen (2012).

2. Signalons par exemple Boehm $(2003,2015)$ et Gravylenko (2013).

3. De nombreuses études d'anthropologie historique ont porté sur le corps, les gestes, les apparences, en particulier en relation avec la question des identités; pour un bilan, voir le supplément 14 des Dialogues d'Histoire ancienne paru en 2015.

4. Voir par exemple Aristote, De anima, II, 2, 413 b5.

5. Le philosophe de Stagire en avait conscience : Heller-Roazen (2011, 25-28). Sur les théories d'Aristote relatives aux sens, voir Sorabji (1971) et Murgier (2013), qui montrent que la hiérarchie sensorielle élaborée par le philosophe dépend en fait de la perspective adoptée. 
possède pas d'organe qui lui soit propre. C'est bien l'ensemble du corps qui sert de récepteur aux stimulations tactiles ${ }^{6}$ - ou plus exactement la peau, qui sert d'interface, rendant ainsi possible le contact entre le sujet percevant et le monde extérieur. Or, cette enveloppe corporelle porte en grec ancien le nom de khrôs, un terme qui met aussi en jeu la notion d'identité et la question de la représentation sociale ${ }^{7}$.

Les articles rassemblés dans ce numéro montrent justement que les modalités de contact entre deux corps varient grandement en fonction de l'identité de celui qui touche et celle de celui qui est touché. L'exemple de la Bible, étudié par Régis Burnet, soulève en particulier une question importante : que se passe-t-il lorsque le corps que l'on touche est d'essence divine? Immédiatement surgit alors un autre problème pour l'helléniste : les dieux possèdent-ils un corps aux yeux des Grecs? En répondant de façon affirmative, Jean-Pierre Vernant (1986) précise qu'il s'agit d'un «sur-corps», à savoir un corps incorruptible, irradiant de lumière, qui creuse ainsi l'écart avec l'enveloppe charnelle des mortels. C'est en effet l'image qui ressort de la tradition littéraire grecque, et ce depuis Homère et Hésiode, considérés par Hérodote comme les pères fondateurs du panthéon hellénique ${ }^{8}$.

En fait, le «sur-corps» des dieux se caractérise surtout par sa polymorphie : les Immortels restent insaisissables et leur apparence se dérobe aux capacités sensorielles humaines ${ }^{9}$. Dans les épopées homériques, ils s'entourent fréquemment d'une nuée ou d'une vapeur épaisse, qui fonctionne comme une cape d'invisibilité. Les mythes soulignent également que, pour un simple mortel, voir une divinité peut aveugler, voire foudroyer, si celle-ci lui apparaît en majesté ${ }^{10}$. Mais il arrive aussi aux dieux de prendre simplement forme humaine, et c'est là qu'il devient alors possible de les toucher, voire de les blesser ${ }^{11}$,

6. Ce point a été souligné notamment par les auteurs de la collection hippocratique : Romeyer-Dherbey $(1991,453)$. Il faut toutefois ajouter que, chez les médecins, la peau n'est pas identifiée comme capteur/récepteur de l'impression tactile : ce sont le doigt et la main qui font office d'organe du toucher : Gravylenko $(2013,27)$.

7. Sur le sens du terme khrôs, «peau, teint», voir Pigeaud (2005).

8. Hérodote, Histoires, II, 53.

9. Pour les dieux homériques, voir Bettini (2016).

10. Les nombreuses études consacrées aux épiphanies des dieux insistent surtout sur l'aspect visuel : par exemple Piettre (2001) et Platt (2011). Cependant, les manifestations physiques de la présence divine passent par toute une gamme de sensations qui engagent d'autres éléments du système perceptif : voir par exemple Burkert (1997); Grand-Clément (2016). 11. C'est le cas de la déesse Aphrodite, atteinte par la lance du héros Diomède alors qu'elle tente de protéger son fils Énée : Iliade, V, 337-343. L'aède souligne alors les limites de l'anthropomorphisme divin : point de sang (baima) qui coule, mais une sirosité nommée ichôr $r$ qui marque la spécificité de la physiologie des Immortels et creuse ainsi l'écart entre hommes et dieux : voir Loraux (1986, 486-491); Ballabriga (1997); Allen-Hornblower (2014). 
ou au contraire de les caresser ${ }^{12}$ - même si l'union d'un mortel avec une déesse peut provoquer la colère des dieux ${ }^{13}$.

L'anthropomorphisme des dieux qui prévaut dans la tradition littéraire fondée par Homère et Hésiode semble donc rendre possible une proximité physique, voire une forme d'intimité entre hommes et dieux, dans les récits qui les mettent en scène. Mais qu'en est-il des pratiques rituelles grecques effectives, qui ont précisément pour objectif d'établir le contact entre le monde terrestre et la sphère divine? Le fidèle peut-il «toucher» le corps des dieux? Et, mieux, cherche-t-il à le faire? On aurait tendance à répondre par la négative, si l'on s'en tient aux actes les plus communs de la religion grecque. En effet, la prière, la libation et le sacrifice sanglant semblent construire une relation sur le mode de la distance : ce sont d'abord les ondes sonores, le flux liquide et les effluves odorants qui servent à activer la communication avec le divin ${ }^{14}$. Mais n'existe-t-il pas des formes de contact plus intimes? C'est là qu'intervient le rôle d'un médiateur, susceptible de rendre possible une relation haptique : l'effigie divine, qui ancre la présence divine dans le territoire de la cité ${ }^{15}$. Certes, chez les Grecs comme chez les Romains, la statue n'est pas nécessaire dans le culte, car le plus important consiste en des gestes accomplis autour de l'autel, en plein air. Pour autant, l'installation (bidrusis) de l'effigie dans son temple permet à la divinité de venir l'habiter, et rend alors sensible - palpable, pourrait-on dire - sa présence, créant un lien plus fort, tangible, avec la communauté des fidèles qui sollicite sa protection et son aide.

Nous allons donc nous intéresser ici à différents types de contacts physiques possibles entre les Grecs et les statues de leurs dieux, afin de déterminer dans quelle mesure ils permettent de «toucher» les dieux euxmêmes. Cela permettra ainsi de percevoir d'éventuelles différences et spécificités par rapport aux modalités ordinaires du toucher. Il faudra en outre garder à l'esprit que les témoignages littéraires, mais aussi épigraphiques et iconographiques que nous mobiliserons nous donnent surtout accès aux

12. L'Hymne bomérique à Aphrodite relate comment Anchise finit par succomber aux charmes de Kypris. Le héros ignore toutefois à qui il a affaire : la déesse s'est en effet donné l'apparence d'une jeune vierge, de façon à ce qu'il n'ait pas peur de faire l'amour avec elle. Ceci dit, la relation intime entre le héros et la divinité n'est évoquée que de manière très allusive dans le poème : aucune caresse n'est explicitement mentionnée. Le contact physique passe en fait par le truchement des ornements et vêtements qu'Anchise enlève les uns après les autres de la peau d'Aphrodite, avant de s'unir à elle : Hymne homérique à Aphrodite, 161-167. 13. Odyssée, V, 93 : «les dieux n'aiment pas voir une déesse avec un homme»; voir aussi Hymne homérique à Aphrodite, 286-289.

14. Sur l'importance des substances odorantes dans les pratiques rituelles grecques, voir notamment Prost (2008).

15. Nous n'entrerons pas dans le débat concernant l'existence ou non d'une catégorie d'effigies divines à part, les «statues de culte» : voir sur ce point Pirenne-Delforge (2008) et Mylonopoulos (2010). 
représentations collectives, c'est-à-dire à la façon dont les Grecs envisagent ce type de relation. Pour mener l'enquête, nous choisirons quelques exemples qui relèvent tantôt de la norme, tantôt de l'exceptionnel et de la déviance.

\section{Oindre, parer et manipuler les statues divines}

Les effigies des dieux conservées à l'intérieur des temples font l'objet de soins particuliers. On sait que, dans plusieurs cités, des fêtes donnent lieu à la manipulation des statues : ces dernières sont lavées, ointes d'huile, vêtues, parées d'ornements ${ }^{16}$. On connaît, à Athènes, le cas des Plyntéries et des Kallyntéries. Chaque année, la vénérable statue en bois d'olivier d'Athéna Polias, conservée sur l'Acropole, est déshabillée, baignée, puis rhabillée et parée. L'effigie est couverte d'un voile le temps durant lequel elle est dépouillée de ses parures. Les deux fêtes vont de pair; accomplies selon un calendrier fixe par de jeunes Athéniennes sélectionnées à cet effet, elles marquent le renouvellement du lien entre la déesse et la polis ${ }^{17}$. Un rituel analogue est pratiqué, dans la même cité d'Athènes, lors des Aphrodisia célébrées en l'honneur d'Aphrodite Pandemos et de Peithô, qui ont un temple sur les flancs de l'Acropole. Une inscription datée de 284 avant notre ère précise en effet que les astynomes doivent veiller à la préparation de la cérémonie, selon la tradition : repeindre les autels et oindre de poix les portes, baigner (lousai) les statues et se procurer une certaine quantité de laine pourpre, sans doute pour le vêtement destiné à revêtir les effigies divines ${ }^{18}$.

Les pratiques rituelles d'entretien (therapeia) des statues sont connues également pour le sanctuaire de Délos, grâce à la riche documentation épigraphique d'époque hellénistique parvenue jusqu'à nous. Les comptes des hiéropes nous renseignent par exemple sur la nature des fournitures nécessaires pour les opérations de kosmèsis, d'epikosmèsis et de kbrusôsis («dorure» : Bourgeois \& Jockey, 2005) qui consistent à faire briller et à orner les effigies divines et leurs demeures ${ }^{19}$. On recourt à des onguents et

16. Linant de Bellefonds (2004); Parker (1990, 27-28).

17. Voir Le Guen Pollet (1991, 29-34) et Brulé (2012).

18. IG, II2, 659. Sur ces différentes fêtes athéniennes, voir Simon (2002, 46-51); pour ce qui concerne plus particulièrement le culte d'Aphrodite Pandémos : Pirenne-Delforge (1994, 26-34).

19. Sur l'epikosmèsis et la ganôsis de la statue en marbre dorée d'Aphrodite, voir Bruneau (1970, 336, 186-187), pour ce qui concerne la dorure et l'epikosmèsis de la statue en bronze d'Artémis). L'auteur pense que, dans le cas des opérations de kosmèsis menées dans l'Héraion de Délos, les soins portent non pas sur l'effigie divine mais sur l'ensemble du sanctuaire. 
à des huiles purifiées ${ }^{20}$, de façon à raviver les couleurs, l'éclat rayonnant et les délicieuses fragrances émanant des effigies divines ${ }^{21}$. Ici, les opérations ne semblent pas avoir été prises en charge par les prêtres et les prêtresses eux-mêmes, mais par un personnel spécifique, les kosmètai, rémunérés pour la mission qui leur est confiée : de telles procédures font donc l'objet d'un contrôle de la part des autorités du sanctuaire (Bruneau, 1970, 253-254).

Comment interpréter ces rituels de parure périodiquement renouvelés, dont on trouve des traces dans d'autres aires culturelles, notamment en Égypte ou en Mésopotamie? Les jeunes Athéniennes en charge des Kallyntéries ont-elles le sentiment, en manipulant l'effigie en bois d'olivier, de procéder à la toilette d'Athéna ou d'être ses chambrières ${ }^{22}$ ? Nos sources ne nous permettent pas de répondre, mais je crois que les rituels de therapeia des images divines visent surtout à capter la présence de la divinité, afin que cette dernière vienne habiter sa statue ou son sanctuaire. Il s'agit d'activer la fonction relationnelle de l'agalma ${ }^{23}$. L'application de fluides lumineux et odorants sur les statues, qui servent de réceptacles tangibles à des puissances supra-humaines invisibles, polymorphes et mobiles, vise ainsi à entretenir le flux de la kharis («grâce») bienfaisante et réjouissante qui relie les dieux aux hommes (Grand-Clément, 2011, 273). Les tissus jouent à cet égard un rôle de premier plan, comme un moyen d'interagir physiquement avec les divinités, selon Cecilie Brøns (2014, 155-157).

L'effigie peut aussi sortir du temple et être manipulée publiquement lors de certains rituels spécifiques ${ }^{24}$. Prenons le cas du culte d'Artémis Orthia à Sparte. Pausanias rapporte que la petite effigie en bois (xoanon) est portée par la prêtresse lors de la flagellation des jeunes gens pratiquée devant l'autel, ce qui permet alors à la déesse de rendre sensible aux participants son implication effective dans la cérémonie. En effet, lorsqu'elle souhaite manifester son mécontentement face au spectacle qui lui est offert, Artémis rend l'objet de plus en plus pesant entre les mains de l'officiante ${ }^{25}$. Une telle tradition existe ailleurs dans le Péloponnèse. En effet, les fouilles archéologiques menées à Messène indiquent qu'il existe un sanctuaire

20. Par exemple en 269 avant notre ère pour la parure (kosmèsis) de la statue de Dionysos : IG, XI, 2, 203; I, 38.

21. Sur l'utilisation de l'huile, voir Leka (2012).

22. Comme ce que l'on observe dans le monde catholique, avec la tradition coutumière d'habiller les statues des Vierges locales, en Espagne : Albert Llorca (2013).

23. Il ne faut donc pas suivre les auteurs chrétiens - en particulier Clément d'Alexandrie dans le livre IV du Protreptique - lorsqu'ils affirment, dans un contexte polémique, que les «païens» croient que le bois, la pierre, le métal sont leurs dieux. Voir Bouffartigue (2007). 24. Sur l'existence de processions qui consistent à prendre la statue de son sanctuaire, à l'apporter aux limites du territoire, puis à la faire réintégrer son emplacement initial, voir Bettinetti (2001, 187-210).

25. Pausanias, Périégèse, III, 16, 11. 
d'Artémis Ortheia où se déroulent des pratiques analogues. Les statues de jeunes femmes qui y ont été découvertes suggèrent en effet l'existence d'un rituel d'initiation incluant la manipulation d'une statuette en bois, sans doute analogue au xoanon laconien ${ }^{26}$.

Les quelques exemples que nous venons d'examiner attestent l'existence de normes rituelles bien établies concernant le traitement des effigies divines, dans le cadre des cultes civiques, mais ils ne résument pas à eux seuls la grande variété des situations et traditions cultuelles locales. Il existe en parallèle des formes de dévotion plus spontanées et individuelles; malheureusement, celles-ci constituent souvent l'angle mort des études sur les religions antiques, en raison des lacunes de notre documentation.

\section{S'adresser à la divinité : la recherche de modes de contact plus intimes}

Nous l'avons dit, les principaux rites grecs supposent une certaine distance. Dans le cas du sacrifice sanglant, il s'agit bien, depuis l'épisode fondateur rapporté par Hésiode, de réaffirmer la coupure radicale qui existe entre les mortels et les Bienheureux, ces derniers se nourrissant simplement du fumet des morceaux brûlés sur l'autel ${ }^{27}$. Quant à la prière, qui peut être réalisée n'importe où, elle ne requiert pas de contact physique avec la statue, l'autel ou tout autre objet censé médiatiser la relation au divin. Elle est généralement effectuée les mains tendues vers le ciel. Un tel geste est interprété comme une façon d'appeler les dieux et de guider vers eux le flux des paroles prononcées à leur intention. Néanmoins, le fait de tendre les mains, paumes ouvertes, pourrait aussi s'apparenter, somme toute, à l'«esquisse d'un contact, un simulacre de toucher», comme le suggère Danièle Aubriot (1992, 406, n. 4).

Il existe quelques cas où un contact physique avec la statue est ouvertement recherché, parce qu'il est susceptible d'offrir un accès personnel privilégié à la divinité : lors d'un rituel de supplication, par exemple. On sait en effet que, en vertu du droit d'asylie, les fugitifs peuvent trouver refuge dans un sanctuaire, pour échapper à leur(s) poursuivant(s) : ils se placent alors sous la protection de la divinité tutélaire du lieu. Dans ce cas, enserrer l'effigie divine confère un surcroît d'immunité par rapport au refuge offert par le lieu sacré, si l'on en croit Plutarque :

26. Celle-ci était placée sur une base à côté de la statue en marbre, fixe, de la déesse, réalisée par Damophon à l'époque hellénistique (voir Themelis, 1994, 122).

27. Hésiode, Théogonie, 559-560. 


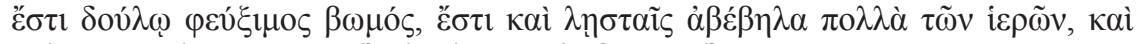

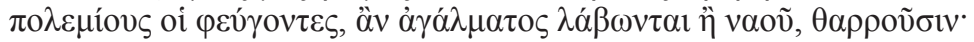

«Un esclave peut se réfugier au pied des autels. Il est des sanctuaires que les voleurs n'osent profaner. Les fuyards sont en sûreté lorsqu'ils ont pu atteindre une statue, ou un temple ${ }^{28}$.»

Plutarque emploie un terme assez générique pour évoquer le geste des suppliants : «saisir» (verbe lambanô) la statue. Signalons que, selon l'une des étymologies proposée pour le mot, le suppliant (biketès) est littéralement «celui qui touche», le radical $h i k$-impliquant la notion de contact physique $^{29}$. Il faut cependant préciser, comme le rappelle Pline (Histoire naturelle, XI, 103), qu'un tel rituel de supplication n'est pas propre aux relations entre les hommes et les dieux : une procédure analogue existe entre deux mortels. Dans ce cas, le suppliant choisit de toucher une partie spécifique du corps de la personne auprès de laquelle il sollicite bienveillance et protection : les genoux ${ }^{30}$. Le geste appuie la demande et active la relation - dissymétrique - qui s'établit alors entre les deux individus ${ }^{31}$. Il s'agit pour le suppliant de capter l'attention et d'émouvoir, grâce au contact établi avec l'une des parties du corps censées contenir l'énergie vitale de la personne sollicitée. Le processus diffère sensiblement lorsque l'on enlace ou que l'on touche les genoux de la statue d'une divinité, au cour d'un sanctuaire. Dans ce cas, le contact communique, comme par capillarité, une forme de sacralité au dévot : c'est bien une part de la puissance de la divinité qui se trouve alors mobilisée.

Dès lors, quiconque ose porter la main sur un suppliant réfugié auprès de la divinité pour implorer sa protection fait preuve d'bubris et d'impiété, et mérite d'être puni par la justice humaine ou divine. Ainsi Ajax, qui s'est rendu coupable du viol de Cassandre, après la prise de Troie, alors que la jeune femme s'était réfugiée au pied de la statue d'Athéna : l'épisode mythologique est devenu un motif iconographique fréquent sur les vases attiques, aux $\mathrm{VI}^{\mathrm{e}}$ et $\mathrm{V}^{\mathrm{e}}$ siècles avant notre ère ${ }^{32}$. Une telle conduite outrageuse, qui semble avoir été plus répandue en temps de guerre, est en général mise sur le compte de l'impiété du barbare, de l'ennemi. Ceci dit, il ne faut pas pour autant imaginer que la norme est toujours respectée par les Grecs eux-mêmes...

28. Plutarque, De la superstition, $166 \mathrm{e}$.

29. Aubriot $(1992,408)$. Chantraine $(1999$, s. v. bikô) souligne que le verbe bikô signifie «arriver, atteindre » et qu'il peut être rapproché du lituanien siékiu, «atteindre avec la main». 30. Il peut s'agir aussi du menton ou des mains : Gould $(1973,96)$.

31. Gould $(1973,96)$. Sur le rite de supplication et ses diverses mises en œuvre rituelles, voir Aubriot (1992, 407-438).

32. Signalons que, dans ce cas, la déesse Athéna n'a pas empêché l'acte : sa vengeance contre le héros n'intervient qu'a posteriori. 
Il existe des cas où les fidèles, lorsqu'ils implorent le secours d'une divinité, quelle qu'en soit la raison, n'hésitent pas, comme les suppliants, à étreindre la statue. Le témoignage de Cicéron fournit un exemple intéressant à propos d'une tradition existant à Agrigente, en Sicile, et dont on ignore l'origine. L'orateur romain évoque en effet une très belle statue d'Héraklès conservée dans un temple près de l'agora, et si vénérée «que sa bouche et son menton sont usés, car dans les prières et les actions de grâce on ne se borne pas à l'adorer, on va jusqu'à l'embrasser (verum etiam osculari solent) ${ }^{33} \gg$. Une forme de dévotion aussi intense, qui abolit la distance habituelle et rappelle le geste du suppliant, pourrait surprendre si l'on songe à l'apparence physique d'une image censée figurer le héros guerrier, à la musculature et à la stature imposantes dans l'iconographie traditionnelle. Pourquoi aller jusqu'à caresser, le gratifier de baisers (verbe osculor) Héraklès? Il s'agit probablement d'une spécificité cultuelle locale : l'Héraklès d'Agrigente est une puissance secourable, analogue à l'Alexikakos pour lequel les Athéniens ont instauré un culte au moment de la grande épidémie qui se développe au début de la guerre du Péloponnèse (Woodford, 1976).

Une autre forme de contact permettant de nouer une relation privilégiée avec les divinités, pour appuyer une prière ou remercier de l'accomplissement d'un vœu, consiste à déposer une offrande au plus près, voire au contact de la statue. La relation est d'autant plus intime lorsqu'il s'agit d'effets personnels, bijoux ou vêtements, que les dévots ont eux-mêmes portés. L'un des cas les mieux connus est celui des offrandes de vêtements effectuées au sanctuaire athénien de Brauron, au $I V^{\mathrm{e}}$ siècle avant notre ère, à l'attention d'Artémis. Des inventaires administratifs affichés sur des stèles dressées sur l'Acropole ont conservé la liste des pièces textiles consacrées à la déesse par les Athéniennes ${ }^{34}$. On y apprend que ces dernières déposent généralement dans le sanctuaire une pièce de leur propre garde-robe ou de celle d'un membre de leur famille, et prennent soin d'y faire figurer leur nom, pour personnaliser leur offrande. Les inscriptions révèlent que certaines de ces étoffes sont placées non pas sur des étagères ou dans des boîtes, mais disposées sur les différentes statues d'Artémis conservées dans le temple. On sait par exemple qu'en 354-344 avant notre ère, la plus ancienne des trois effigies de la déesse (sans doute une statue acrolithe ou en bois, représentant la déesse assise sur un trône) est recouverte d'au

33. Cicéron, Seconde action contre Verrès, IV, 94 (trad. G. Rabaud, CUF). La statue avait eu le malheur de susciter la convoitise de l'insatiable Verrès, qui ne put cependant la posséder, puisque ses sbires durent battre en retraite face à l'animosité des Agrigentins soucieux de conserver leur Héraklès.

34. Les six stèles découvertes sur l'Acropole d'Athènes (IG, II2, 1514-1525; 1528-1531) ont été éditées par Cleland (2005). 
moins quatre étoffes : deux châles fins, l'un au nom de Théanô, l'autre de Pentetèris, un vêtement en laine de Tarente, toujours au nom de Théanô, et un tissu brodé ${ }^{35}$. Les inventaires dressés par les magistrats ne fournissent malheureusement aucune précision quant aux circonstances dans lesquelles le tissu a été placé directement au contact de l'effigie divine. On ignore donc en vertu de quoi lesdites Pentétéris et Théanô ont obtenu le droit de revêtir la statue de leurs offrandes. L'une des hypothèses que l'on peut formuler consiste à voir dans ces deux femmes non pas de simples particulières, mais des prêtresses ayant accompli un dépôt au nom de l'ensemble de la communauté.

Une telle interprétation pourrait être étayée par un épisode du chant VI de l'Iliade. Les Troyennes, menées par Hécube, décident de se rendre dans le temple d'Athéna pour implorer l'aide de la déesse. La reine choisit de lui offrir un tissu richement décoré et sélectionne donc dans les réserves du palais, le plus beau et le plus brillant des voiles multicolores ${ }^{36}$. Mais une fois dans le temple, c'est la prêtresse Théanô qui prend le relais. Seule, elle accomplit le geste d'offrande et prononce la prière au nom de l'ensemble des Troyennes :

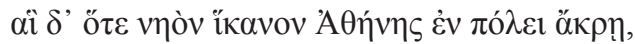

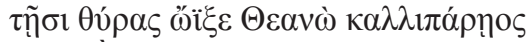

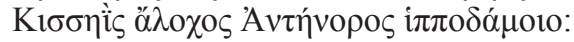

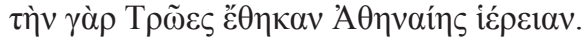

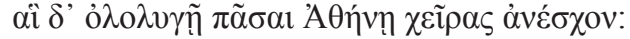

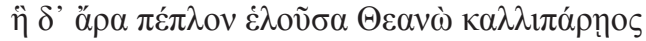

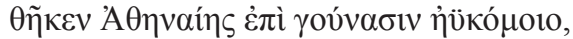

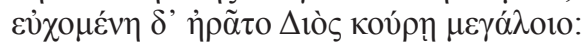

«À peine ont-elles atteint le temple d'Athéna, en haut de l'acropole, que les portes leur en sont ouvertes par la Theanô aux belles joues, fille de Cissès, épouse d'Anténor, le dompteur de cavales, que les Troyens ont fait prêtresse d'Athéna. Avec le cri rituel, vers Athéna toutes tendent les bras. Théanô aux belles joues prend le voile, le dépose sur les genoux d'Athéna à la belle chevelure, puis, suppliante, elle adresse ce vœu à la fille du grand Zeus ${ }^{37}$.»

On constate que la contribution des Troyennes et de la reine au rituel de supplication se limite, dans le temple, à adopter l'attitude de l'orant et à garder une distance respectueuse, tandis que la maîtrise de la parole et l'accès à la statue, deux voies de communication plus directes avec Athéna,

35. $I G, \mathrm{II}^{2}, 1514,35-38$. Voir Brøns (2014, 133-135).

36. Iliade, VI, 287-295.

37. Iliade, VI, 297-304 (trad. P. Mazon, CUF). Soulignons que le passage décrit ici ne trouve aucun équivalent dans le reste des poèmes homériques; c'est la seule fois où une statue divine est mentionnée et l'on notera d'ailleurs que le poète n'emploie pas de terme spécifique, puisqu'il est simplement question d'«Athéna ». 
demeurent l'apanage de la prêtresse, qui fait ainsi figure de médiatrice privilégiée ${ }^{38}$. Cet exemple, puisé dans la tradition littéraire, invite à s'interroger sur les conditions réelles d'accès aux statues divines, dans les sanctuaires grecs, à partir de l'époque archaïque.

\section{Accéder ou ne pas accéder à la statue, telle est la question}

Il n'existe pas de norme générale et universelle réglementant l'accès aux statues des dieux dans le monde grec : là encore, la diversité règne en matière d'usages et dépend de facteurs liés aux traditions religieuses locales et à la configuration du lieu où se trouve conservée ladite statue (un temple, un enclos, une grotte... $)^{39}$.

Le droit de toucher ou non la statue divine n'a pourtant rien d'anodin. Il engage un certain nombre de notions importantes, en particulier la question de la pureté, comme en témoigne une tragédie d'Euripide, représentée à Athènes à la fin du $\mathrm{V}^{\mathrm{e}}$ siècle avant notre ère : Iphigénie en Tauride. La pièce, dont l'intrigue se déroule dans le royaume barbare du roi Thoas, relate le vol réussi (et légitime!) de la statue d'Artémis par Iphigénie et Oreste. Un passage de la tragédie indique clairement que seule la jeune femme, parce qu'elle est la desservante officielle du culte, a la possibilité de toucher l'image sacrée :

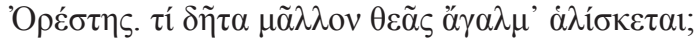

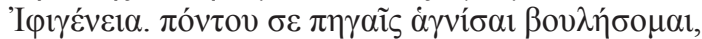

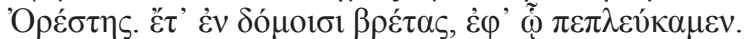

'I

«Oreste. Comment nous sera-t-il plus facile de ravir la statue d'Artémis?

Iphigénie. J'annoncerai l'intention de te purifier dans l'eau de mer.

O. Mais la statue, but de notre voyage, est encore dans le temple...

I. Je dirai qu'il faut la purifier, elle aussi, parce que tu l'as touchée.» $[\ldots]$

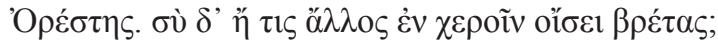

'I

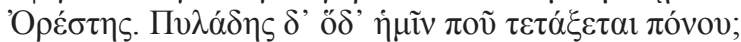

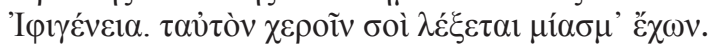

38. Sur la fonction de médiation des prêtres et prêtresses dans le monde grec, voir PirenneDelforge (2010).

39. Sur les questions d'accès à la statue et au temple par les fidèles, voir par exemple Corbett (1970) et Blidstein (2015). 
«O. Est-ce toi-même ou quelqu'un d'autre qui portera la statue dans ses mains?

I. Moi : car je suis la seule à pouvoir y toucher.

O. Et quel rôle Pylade jouera-t-il dans notre entreprise?

I. On dira que ses mains sont souillées comme les tiennes ${ }^{40}$.»

Iphigénie invoque une norme rituelle (bosion esti) selon laquelle seule la prêtresse peut porter la main sur l'effigie ${ }^{41}$. Notons qu'Euripide emploie ici au moins trois verbes différents pour évoquer le contact physique. Le premier, haliskomai, «saisir», renvoie ordinairement à la prise de guerre; thigganô possède le sens plus général de toucher de la main, et s'applique également au domaine érotique et aux relations sexuelles; enfin, phérô, comme chez Pausanias, désigne l'action de «porter, transporter». Il n'existe donc pas de vocabulaire spécialisé dans le domaine rituel pour évoquer le contact avec l'image divine.

Le dialogue entre Iphigénie et Oreste mobilise la notion de miasma, qui renvoie à des formes de souillure à la fois symbolique et matérielle, susceptibles de perturber la bonne communication avec les dieux. C'est donc pour éviter tout risque de contamination qu'il faut maintenir un contrôle strict de l'accès à la statue. Le personnel autorisé est censé respecter scrupuleusement les exigences de pureté rituelle, c'est-à-dire être en état d'bagneia ${ }^{42}$.

On s'étonnera alors du fait que la documentation épigraphique, qui comporte tant de prescriptions relatives à l'accoutrement ou à l'état de pureté des fidèles pénétrant dans un sanctuaire, ne fournisse aucune référence explicite au comportement à adopter vis-à-vis de la statue de la divinité ${ }^{43}$. Certes, quelques rares témoignages épigraphiques d'époque hellénistique ou romaine indiquent que, lors des fêtes publiques, seuls les desservants du culte peuvent toucher les objets sacrés, l'autel et l'effigie divine ${ }^{44}$, mais il est impossible de déterminer s'il s'agit d'une norme répandue. Une telle discrétion de la documentation épigraphique - remarquable au regard du nombre d'inscriptions interdisant par exemple de couper les arbres de

40. Euripide, Iphigénie en Tauride, 1038-1047 (trad. personnelle).

41. La statue n'est pas la seule chose sacrée susceptible de faire l'objet d'un tel interdit: Thucydide mentionne par exemple une source en Béotie que l'on «ne pouvait toucher», excepté à des fins rituelles : Histoire de la guerre du Péloponnèse, IV, 97, 3.

42. Rappelons qu'un tel souci de pureté rituelle anime la plupart des normes rituelles grecques; sur la notion d'hagneia, voir Parker (1990, 147-150).

43. Parker (1990, 176-180).

44. Par exemple la loi concernant le sanctuaire du Thesmophorion au Pirée, d'après une inscription de la seconde moitié du $\mathrm{IV}^{\mathrm{e}}$ siècle avant notre ère (les fidèles ne peuvent faire des purifications, déposer des objets sacrés ou s'approcher de l'autel en l'absence de la prêtresse) : Le Guen-Pollet (1991, 15-19). Sur la question de l'accès aux statues dans le monde romain, voir Estienne $(2001,207)$. 
l'alsos - ne signifie pas que la question importe peu aux yeux des Grecs. Il est possible qu'une telle réglementation ne soit pas nécessaire, en raison du contexte d'exposition de la statue. Rappelons qu'elle se trouve généralement placée à l'intérieur du temple, dont les portes ne sont pas ouvertes en permanence. L'accès à l'intérieur de l'édifice fait l'objet d'un contrôle; ce sont les prêtres et les prêtresses qui en possèdent la clef.

De plus, les données archéologiques rassemblées et analysées par Sophie Montel indiquent que le dispositif architectural peut concourir à restreindre les velléités des fidèles et induire de fait une normalisation des comportements. La base et les traces des aménagements afférents à l'installation des statues (qui, elles, ont souvent disparu) dans les sanctuaires demeurent encore visibles. Or, ces vestiges révèlent qu'entre l'époque archaïque et l'époque hellénistique, la scénographie a évolué et de nouveaux dispositifs architecturaux ont vu le jour dans les sanctuaires pour isoler la statue placée au fond de la cella du temple : monumentalisation et élévation du socle, érection de barrières... Dans la Crète d'époque géométrique, il semblerait que l'on pouvait saisir les effigies divines, de petites dimensions, posées sur des banquettes comme l'indique le dispositif mis au jour à Dréros. Mais par la suite, l'apparition et la diffusion de statues monumentales (comme celles de Phidias) ont contribué, dans certains cas, à instaurer une distance entre le dévot et l'image divine, une distance matérialisée physiquement par la présence de grilles, comme à Andanie (Montel, 2008).

S'il ne faut nullement en conclure à une évolution générale de la religion grecque, marquée par un éloignement progressif des dieux, il n'en reste pas moins que les effets de mise en scène conditionnent le rapport qui se noue entre le fidèle et la divinité, dans la mesure où ils rendent possible - ou non - un accès direct à la statue. Pausanias, qui a visité de nombreux sanctuaires grecs au $\mathrm{II}^{\mathrm{e}}$ siècle de notre ère, mentionne quelques cas intéressants où ce n'est pas le dévot qui est soumis à restriction, mais l'effigie elle-même, car elle est entravée par des liens ou des chaînes. De tels dispositifs pourraient s'expliquer par l'agency et le pouvoir que les Grecs reconnaissaient à certaines effigies sculptées, jugées très anciennes et attribuées à des artisans mythiques virtuoses comme Dédale ${ }^{45}$. Parce qu'elles semblaient vivantes, on souhaitait les empêcher de se mouvoir. Mais, nous allons le voir, la présence d'entraves procède parfois d'une

45. Ainsi, la très ancienne statue de Déméter que le périégète a pu voir dans un temple en terre crue de Phocide (Pausanias, Périégèse, X, 35, 10). Sur la tradition des œuvres dédaliques dotées d'animation, voir Frontisi-Ducroux (1975, 102-104), et plus largement sur l'agency des statues grecques, voir Bremmer (2013) - le travail de Freedberg (1998) reste à cet égard une référence. 
volonté d'exercer une emprise non pas sur l'objet matériel, animé, mais bien sur la puissance divine concernée ${ }^{46}$.

\section{Les statues enchaînées chez Pausanias}

Lorsqu'il a visité la Laconie, Pausanias a été intrigué en voyant une statue d'Enyalios, dieu de la guerre, qui portait des fers aux pieds (Périégèse, III, 15, 7). Les Lacédémoniens lui ont fourni l'explication suivante : un tel dispositif visait à empêcher le dieu d'abandonner le territoire civique. La littérature gréco-romaine renferme en effet des cas de divinités qui quittent la cité dont ils ont la charge, lors d'un conflit, soit que leur bienveillance ait été captée par les ennemis, soit qu'elles-mêmes aient décidé, sous le coup de la colère, de se détourner de ceux qu'elles considèrent comme de futurs vaincus. Les Laconiens estimaient donc qu'en attachant l'effigie sculptée, ils pourraient retenir prisonnier le dieu lui-même, comme ils l'auraient fait avec un soldat, pour l'empêcher de déserter ${ }^{47}$.

Comme Pausanias n'exprime aucun jugement personnel sur l'étiologie fournie par les Spartiates, il est difficile de savoir s'il l'estime recevable ou fantaisiste. En revanche, il n'hésite pas à se moquer ouvertement d'une autre tradition locale - toujours laconienne - entourant la statue d'Aphrodite Morphô, conservée selon ses dires au premier étage d'un temple très ancien. Il s'agit d'une petite statue de bois représentant la déesse assise, voilée, avec des fers aux pieds, et que l'on disait avoir été dédiée par le roi Tyndare. Pausanias rapporte au sujet de sa consécration deux étiologies recueillies sur place :

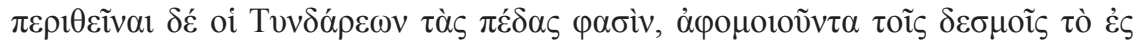

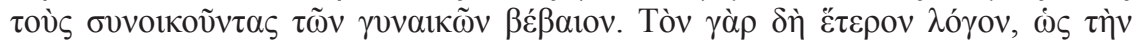

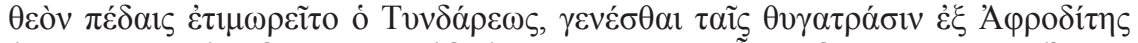

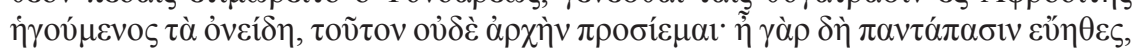

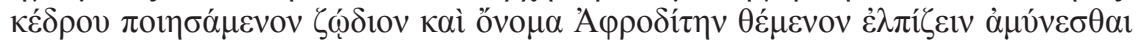

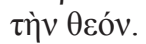

«On dit que Tyndare lui mit ces fers, comme un symbole de l'attachement que les femmes doivent avoir pour leurs maris. Suivant d'autres, il enchaîna cette déesse pour se venger, s'en prenant à elle de la conduite honteuse de ses filles; mais cette dernière tradition ne me paraît mériter aucune foi : il aurait

46. C'est clairement le cas de la statue en bronze d'Actéon réalisé par les Orchoméniens sur les conseils de l'oracle de Delphes : on l'avait attachée à un rocher afin d'éviter que son spectre ne vienne semer le trouble dans la cité (Pausanias, Périégèse, IX, 38, 5).

47. On retrouve un motif analogue lors du siège de Tyr par Alexandre : les historiens grecs rapportent que les habitants de la ville enchaînèrent la statue d'Apollon à celle de Melqart pour empêcher une trahison du dieu grec (voir plus bas). 
été en effet bien stupide s'il avait cru qu'en faisant une statue de cèdre, et en lui donnant le nom d'Aphrodite, il pouvait se venger de la déesse elle-même ${ }^{48}$.»

Pausanias ne commente pas la première explication fournie par les Lacédémoniens (à savoir une stratégie iconographique, les entraves devenant une sorte d'attribut divin fonctionnel, le signe de la fidélité conjugale), mais il récuse clairement la seconde. Il refuse en effet d'y voir le témoignage d'une punition ${ }^{49}$ que Tyndare aurait voulu infliger à la déesse en raison de l'infidélité de ses filles : il serait ridicule selon lui de confondre l'effigie en bois et la déesse ${ }^{50}$.

Il n'y a que des enfants pour croire qu'étrangler une statue d'Artémis revient à étrangler Artémis elle-même - mais cela relève alors du jeu : c'est ce que révèle une anecdote rapportée par Pausanias, au sujet d'un village d'Arcadie, Condylée. L'auteur raconte l'histoire tragique à l'origine de l'épiclèse Apanchomené («la Pendue») sous laquelle y est vénérée Artémis. Les habitants de Condylée décidèrent de lapider les enfants qui s'étaient amusés à pendre l'effigie de la déesse :

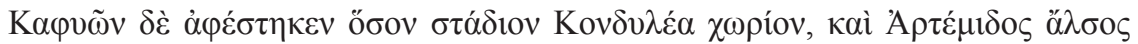

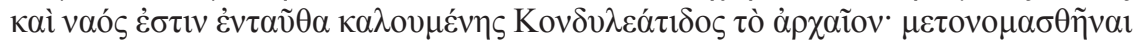

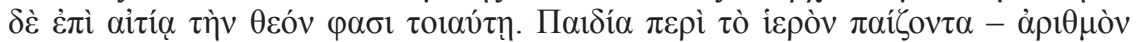

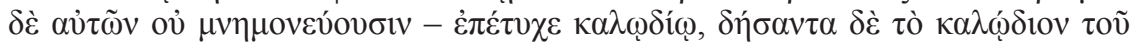

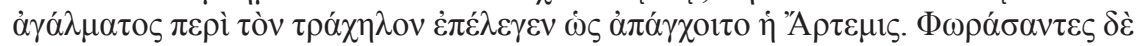

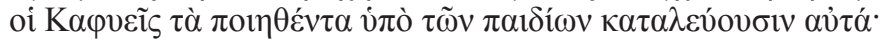

«Distant de Kaphyai d'environ un stade se trouve le site de Kondyléa, et il y a là un bois sacré et un temple consacré à une Artémis anciennement appelée Kondyléatis. Si la déesse changea d'épiclèse, c'est, dit-on, pour la raison suivante. Des enfants qui jouaient autour du sanctuaire (leur nombre n'a pas été retenu) trouvèrent par hasard une cordelette et, après avoir noué cette cordelette autour du cou de la déesse, ils disaient qu'Artémis était pendue. Les gens de Kaphyai, ayant découvert ce que les enfants avaient fait, les lapidèrent ${ }^{51}$.»

En agissant ainsi, les habitants de Condylée espéraient éviter le déclenchement de la colère divine. Pour eux, porter atteinte physiquement à l'effigie revenait à s'en prendre d'une façon ou d'une autre à la divinité elle-même. Si le récit de Pausanias s'arrêtait là, on en déduirait que les enfants reçurent un châtiment mérité, cautionné par Artémis elle-même, victime de l'offense. Pourtant, la suite de l'histoire nous apprend que, si la

48. Pausanias, Périégèse, III, 15, 11.

49. Le verbe employé, атпио, exprime clairement une forme de contrainte : il sert à définir par exemple les actes de rétorsion à l'encontre d'un adversaire.

50. Sur l'attitude de Pausanias par rapport à ce type de «naïveté», voir Pirenne-Delforge (2008b, 73).

51. Pausanias, Périégèse, VIII, 23, 6-7 (trad. M. Jost, CUF). 
déesse se mit finalement en colère, ce ne fut pas à l'encontre de ses jeunes «bourreaux», mais à cause de la réaction excessive des adultes. Artémis, divinité courotrophe, prit en effet le parti des enfants et se vengea en faisant accoucher les femmes d'enfants morts-nés. L'épidémie conduisit finalement les habitants à consulter l'oracle de Delphes : pour expier leur faute, ils durent donner aux enfants une sépulture, instaurer un sacrifice expiatoire annuel pour eux, et changer l'épiclèse de la déesse. L'anecdote est intéressante à plus d'un titre : elle confirme que, dans ce cas, toucher la statue d'un dieu ne signifie pas affecter physiquement le dieu - Artémis n'a pas souffert, contrairement à l'Aphrodite d'Homère blessée par Diomède, mais elle montre aussi que les puissances divines suivent avec attention le sort des effigies que les mortels leur consacrent.

\section{Quand les dévots vont trop loin... Entre violences et caresses}

Parce qu'elles possèdent une valeur matérielle et symbolique forte, les statues des dieux peuvent également faire l'objet de dégradations et de prédations ${ }^{52}$. Il n'y a pas lieu de revenir sur les multiples cas de godnapping ${ }^{53}$ recensés dans la tradition littéraire gréco-romaine : ils s'inscrivent généralement dans un contexte de guerre ${ }^{54}$. Le paradigme mythique est le vol du Palladion par Ulysse et Diomède qui, dans l'Enéide, est présenté comme une profanation provoquant la fureur d'Athéna, indignée par « le rapt de la sainte effigie, où de leurs mains sanglantes ils ont osé toucher les bandelettes virginales de la déesse» («corripuere sacram effigiem, manibusque cruentis/virgineas ausi divae contingere vittas $\gg)^{55}$. On connaît en Mésopotamie et au Proche-Orient de nombreux cas de captures et de déportations de statues divines, depuis le III ${ }^{\mathrm{e}}$ millénaire avant notre ère, avec le développement des grands empires. Il s'agit par exemple d'une pratique de guerre habituelle chez les néo-Assyriens, au gré de leurs conquêtes : les dieux des vaincus font partie du butin convoyé suite aux victoires. Elle n'est pas tant présentée comme un châtiment infligé par le roi victorieux que comme la marque de la désertion des puissances divines, qui se détournent des peuples soumis; il s'agit de marquer un transfert de légitimité et le déplacement du lieu du pouvoir (Holloway, 2001).

52. Nous ne traiterons ici que des cas où l'intégrité de la statue a été préservée et n'aborderons donc pas le riche dossier des mutilations et destructions dont ont pu être victimes les images divines, et que l'on pourrait rattacher aux cas d'outrages aux statues honorifiques (voir Azoulay, 2009).

53. La formule est de l'assyriologue Alasdair Livingstone (1997, 168).

54. Sur l'histoire de ces prédations et les enjeux qui les sous-tendent, voir Miles (2008).

55. Virgile, Énéide, II, 168-169 (trad. J. Perrete, CUF). 
Les Grecs ont été confrontés à de nombreuses reprises au godnapping. Le plus souvent, la tradition historiographique hellénique présente un tel comportement comme étant le fait des Barbares ennemis : les Perses lors des guerres médiques ${ }^{56}$, ou encore les Carthaginois lors des guerres de Sicile. Les prédations opérées par les Romains lors de leurs conquêtes prennent en revanche une forme différente : il s'agit souvent de collecter des œuvres d'art grecques, plutôt que de capter des puissances divines. Les temples de Rome deviennent ainsi peu à peu de véritables galeries d'art. Plutarque rapporte que la tradition d'y dédier des statues prises à l'ennemi remonte à la prise de Syracuse par Marcellus, en 212 avant notre ère. Mais d'autres traditions présentent au contraire le général comme fort respectueux du patrimoine grec (Rutledge, 2012, 36-38). La pratique romaine, justifiée par Strabon, semble se généraliser à partir de César, donnant alors naissance aux grandes collections de sculptures grecques, qui peuvent être considérées comme étant à l'origine des premiers musées ${ }^{57}$.

Il est plus difficile d'interpréter les actions de godnapping commises par les Perses, les Phéniciens ou les Carthaginois, car nous ne disposons que du témoignage des Grecs. Pour ces derniers, le rapt des statues des dieux avait une coloration plus religieuse que politique : ils y voyaient la marque de l'impiété (asebeia) et de la superstition (deisidaimônia) des Barbares (Bonnet \& Grand-Clément, 2013). Il semblerait que l'idée de capter la puissance des dieux du camp adverse ait joué, puisque l'on connaît plusieurs cas de statues grecques enlevées à des cités de Sicile et installées à Carthage pour faire l'objet d'un culte ${ }^{58}$. Un Apollon en bronze, colossal, pris à la cité de Géla, fut même envoyé à Tyr, où il se trouvait lorsqu'Alexandre le Grand, en 331 avant notre ère, entreprit le siège de la ville. Selon Diodore, Plutarque et Quinte-Curce ${ }^{59}$, les Tyriens, effrayés à l'idée qu'Apollon ne les abandonnât pour rejoindre le camp de l'ennemi, attachèrent sa statue avec des chaînes d'or à son piédestal ou, selon une autre version, à l'autel du dieu tutélaire de la cité, Melqart (identifié par les auteurs grecs comme Héraklès). Pour évoquer la contrainte opérée sur la statue par les Tyriens, Diodore emploie le verbe kathubrizô, un terme significatif, puisqu'il renvoie à une action violente et peut aussi, dans le cas d'un contact physique, désigner le viol.

Le gouverneur Verrès, rendu célèbre par les Verrines de Cicéron, a un parfait profil de godnapper - même s'il ne s'en prend pas seulement aux

56. Citons le cas du groupe statuaire en bronze des tyrannoctones réalisé par Anténor et placé sur l'Agora, qui a été emporté à Suse par Xerxès (Arrien, Anabases, III, 16, 3).

57. Rutledge (2012, 123-157) souligne l'importance politique de ces butins de guerre, à replacer dans un contexte de forte compétition sociale.

58. Par exemple l'Artémis de Ségeste (Cicéron, Seconde action contre Verrès, IV, 72).

59. Diodore, XVII, 41, 7-8; Quinte-Curce, IV, 3, 21-23; Plutarque, Alexandre, 24. Voir Bonnet (2015, 83-86). 
effigies des dieux. Ses motivations sont complexes ${ }^{60}$; il est animé par un désir de posséder, qui ne s'accompagne pas nécessairement d'une volonté de toucher. Mais dans la bouche de Cicéron, l'action du gouverneur se colore d'impiété et engage clairement la dimension tactile. L'orateur va en effet jusqu'à assimiler à un viol le rapt d'une statue de Déméter, prise aux habitants d'Henna : «C'est à Henna que tu osais, toi, enlever une statue de Cérès; c'est à Henna que tu t'es efforcé, toi, de tirer brusquement la Victoire de la main de Cérès et de ravir une déesse à une déesse? Et cependant l'audace de les outrager (violare), d'y toucher (attingere) ne s'est jamais trouvée chez des hommes bien plus près d'être des criminels que des personnes religieuses ${ }^{61}$.»

Les paroles accusatoires de Cicéron rappellent que certaines statues divines peuvent susciter le désir. Le cas d'agalmatophilie le plus connu ${ }^{62}$ est sans contexte celui qui concerne l'Aphrodite de Cnide, une sculpture en marbre réalisée dans les années 350 avant notre ère par Praxitèle ${ }^{63}$. Cette œuvre en faisait, selon le témoignage de Pline ${ }^{64}$, la fierté de la cité et générait un tourisme lucratif. Sa renommée tenait au fait que le sculpteur avait innové, en représentant pour la première fois la déesse dénudée, se préparant au bain ou en sortant. Une tradition ancienne rapportée par Athénée ${ }^{65}$ affirme que Praxitèle avait pris pour modèle une courtisane célèbre, Phryné, qui se serait déshabillée en public avant de plonger dans la mer, lors d'une fête en l'honneur de Poséidon. La statue originale n'a pas été conservée, mais il est possible d'en connaître l'apparence grâce aux copies réalisées à l'époque romaine ${ }^{66}$. Aphrodite faisait de la main droite le geste de se cacher le sexe et tenait de la main gauche un vêtement. La peau de la déesse se trouvait ainsi offerte au regard - et pouvait susciter la volonté de toucher. Le matériau - un marbre d'excellente qualité, d'après nos sources - employé pour suggérer le velouté de la carnation, ainsi que le traitement de surface - certainement un enduit de cire destiné à faire briller la pierre - contribuaient à stimuler ce que nous pourrions appeler l' «imagination tactile» du spectateur et à faire naître en lui une pulsion érotique.

Un passage du pseudo-Lucien, auteur satirique du début du $\mathrm{IV}^{\mathrm{e}}$ siècle de notre ère, nous renseigne sur le dispositif choisi par les Cnidiens pour

60. Sur les motivations de Verrès, voir Robert (2007, p. 15-34); Miles (2008, 105-151).

61. Cicéron, Seconde Action contre Verrès, IV, 112 (trad. CUF).

62. Sur l'agalmatophilie, voir notamment Gourevitch (1982).

63. Sur cette statue, voir Stewart (1997, 97-106).

64. Pline, Histoire naturelle, XXXVI, 20-21.

65. Athénée, Deipnosophistes, XIII, 590.

66. Au moins deux cents copies, réalisées dans différents médias, ont été recensées par Seaman (2004) pour reconstituer l'apparence originelle de la statue. 
mettre en scène l'œuvre de Praxitèle ${ }^{67}$. Le temple comportait deux entrées, l'une, principale, et l'autre, au fond, offrant un point de vue privilégié sur la partie arrière de la statue. Les visites étaient contrôlées et le temple fermé à clef la nuit. On ne pouvait faire le tour de la statue, mais il semblerait que l'un des visiteurs ait réussi, en tordant le cou, à déposer un baiser sur la joue de la statue ${ }^{68}$ : un accès direct était donc possible, en dépit des restrictions mises en place. Une célèbre anecdote racontée à Cnide suggère même qu'un jeune homme, éperdument amoureux de la statue, aurait osé aller plus loin : déjouant la surveillance des prêtresses, il se serait fait enfermer dans le temple afin de passer la nuit avec la statue. On disait que son sperme avait laissé une empreinte indélébile : une trace entachait la cuisse de la déesse, et c'est précisément pour en expliquer l'origine que l'on racontait l'histoire ${ }^{69}$.

L'affaire de l'amant de l'Aphrodite de $\mathrm{Cnide}^{70}$, qu'elle soit fictive ou véridique, renvoie à un paradigme mythique : l'histoire de Pygmalion, connue par la version qu'en a laissée Ovide dans le livre X de ses Métamorphoses. Il existe cependant au moins deux différences notables. D'une part, la statue qui suscite le désir du sculpteur chypriote est celle d'une jeune vierge, et non pas celle d'Aphrodite; cette dernière agit en revanche dans le poème en tant que puissance divine, capable d'animer l'effigie d'ivoire. D'autre part, l'effigie adulée est en ivoire et non en marbre. Ovide insiste dans son poème sur la relation charnelle et tactile qui manifeste le désir du sculpteur pour la statue qu'il a lui-même façonnée :

Saepe manus operi temptantes admouet, an sit

Corpus an illud ebur; nec adhur ebur esse fatetur.

Oscula dat reddique putat loquiturque tenetque

Et credit tactis digitos insidere membris

Et metuit, pressos ueniat ne liuor in artus [...].

«Souvent, il approche ses mains du chef-d'œuvre pour s'assurer si c'est là de la chair ou de l'ivoire et il ne peut encore convenir que ce soit de l'ivoire. Il donne des baisers à sa statue et il s'imagine qu'elle les rend; il lui parle, il la serre dans ses bras; il se figure que la chair cède au contact de ses doigts et il craint qu'ils ne laissent une empreinte livide sur les membres qu'ils ont pressés $[\ldots]^{71}$.»

67. Pline l'Ancien livre une autre description; pour un essai de reconstitution du dispositif, voir Montel (2010).

68. Pseudo-Lucien, Les Amours, 13.

69. Pline, Histoire naturelle, XXXVI, 21. Voir aussi Lucien, Imagines, 4.

70. Sur l'existence d'anecdotes analogues : Rutledge (2012, 113-115).

71. Ovide, Métamorphoses, X, 254-258 (trad. G. Lafaye, CUF). 
Et c'est encore par les sensations tactiles, et non visuelles, que le sculpteur chypriote réalise que la statue prend vie, après le baiser qu'il lui a donné :

Admouet os iteum, manibus quoque pectora temptat;

Temptatum mollescit ebur positoque rigore

Subsidit digitis ceditque, ut Hymettia sole

Cera remollescit tractataque pollice multas

Flectitur in facies ipsoque fit utilis usu.

Dum stupet et dubie gaudet fallique ueretur,

Rursus amans rursusque manu sua uota retractat;

Corpus erat; saliunt temptatae pollice uenae.

«De nouveau il en approche sa bouche, tandis que ses mains tâtent la poitrine; à ce contact, l'ivoire s'attendrit; il perd sa dureté, il fléchit sous les doigts; il cède; ainsi la cire de l'Hymette s'amollit au soleil [...]. Sa main palpe et palpe encore l'objet de ses désirs; c'était bien un corps vivant : il sent des veines palpiter au contact de son pouce ${ }^{72}$.»

Le toucher joue un rôle ici essentiel : Ovide insiste sur le geste quasi médical de Pygmalion qui, tel un médecin, ausculte et constate que le corps qu'il palpe est bien vivant.

\section{Conclusion}

L'éventail des formes de contacts possibles entre les Grecs et les statues de leurs dieux est large, nous l'avons constaté : de la toilette d'entretien aux étreintes des suppliants, de l'enchaînement aux déportations forcées, des caresses aux baisers enflammés, la gamme des attitudes et les gestes oscille entre dévotion, superstition et impiété. Les façons de toucher contribuent ainsi à définir et redéfinir sans cesse les frontières entre ce qu'il faut faire et ce qu'il ne faut pas faire, pour entrer correctement en communication avec les dieux. Un même geste peut avoir deux visées bien différentes, selon le contexte : embrasser l'Héraklès d'Agrigente ne procède pas de la même intentionnalité que le baiser volé à l'Aphrodite de Cnide.

Parfois, le contact physique avec l'image du dieu est perçu comme une voie d'accès privilégié au monde divin, qui acquiert par là une épaisseur tangible - et le fidèle a ainsi l'impression de «toucher» le dieu. Mais parfois la puissance divine se dérobe à tout contact effectif et rappelle au fidèle le caractère irréductible de la barrière qui sépare les Immortels des hommes. En fait, la relation haptique établie avec l'effigie divine diffère de celle qui existe ordinairement entre les êtres humains, car la première est

72. Ovide, Métamorphoses, X, 282-289 (trad. G. Lafaye, CUF). Voir Stoichita (2008). 
profondément dissymétrique, tandis que la seconde repose sur la réciprocité. Lorsque le dieu touche, c'est pour manifester son pouvoir et opérer une transformation du corps humain ${ }^{73}$, tandis qu'un mortel ne possède pas la faculté, même s'il tente d'atteindre le dieu à travers sa statue, d'agir sur la nature divine. Cela confirme que, dans le système polythéiste grec, comme le soulignait Jean-Pierre Vernant, les dieux sont des puissances, et non des personnes.

\section{BIBLIOGRAPHIE}

ALBERT LLORCA Marlène, «Les statues habillées dans le catholicisme», Archives de sciences sociales des religions, 164, 2013, p. 11-23.

ALLEN-HORNBLOWER Emily, «Gods in Pain: Walking the Line between Divine and Mortal in Iliad 5», Lexis: Rivista di poetica, retorica e comunicazione nella tradizione classica, 32, 2014, p. 27-57.

AUBRIOT-SÉVIN Danièle, Prière et conceptions religieuses en Grèce ancienne jusqu'à la fin du $V^{e}$ siècle av. F.-C., Lyon, Maison de l'Orient méditerranéen, 1992.

AZOULAY Vincent, «La gloire et l'outrage. Heurs et malheurs des statues honorifiques de Démétrios de Phalère», Annales. Histoire, Sciences Sociales, 2009 (2), p. 303-340.

BALLABRIGA Alain, «La nourriture des dieux et le parfum des déesses», Mètis, 12, 1997, p. 119-127.

BAUMER Lorenzo, «Où le dieu touche. Réflexions archéologiques sur les sanctuaires d'Asclépios», dans $\mathrm{Ph}$. Borgeaud \& D. Fabiano (éd.), Perception et construction du divin dans l'Antiquité, Genève, Droz, 2013, p. 147-164.

BETTINETTI Simona, La statua di culto nella pratica rituale greca, Bari, Levante, 2001.

BETTINI Maurizio, Visibilità, invisibilità e identità degli dèi, dans C. Bonnet \& G. Pironti (éd.), Gli dèi di Omero. Politeismo e poesia in Grecia antica, Rome, Carocci, 2016, p. 29-57.

BLIDSTEIN Moshe, «Entering a Sanctuary the Wrong Way», Scripta Classica Israelica, 34, 2015, p. 165-181.

BOEHM Isabelle, «Toucher du doigt : le vocabulaire du toucher dans les textes médicaux grecs et latins», dans Manus medica. Actions et gestes de l'officiant dans les textes médicaux latins. Questions de thérapeutique et de lexique, Aix-en-Provence, Publications de l'université de Provence, 2003, p. 229-240.

73. Voir l'article de Baumer (2013) sur les sanctuaires de guérison et intitulé «Où le dieu touche». 
BONNET Corinne, Les enfants de Cadmos, Paris, De Boccard, 2015.

— \& GRAND-CLÉMENT Adeline, «Quand les statues divines se meuvent et (s')émeuvent, entre Grecs et Barbares», dans Ph. Borgeaud $\&$ D. Fabiano (dir.), Perception et construction du divin dans l'Antiquité, Genève, Droz, 2013, p. 35-59.

BOUFFARTIGUE Jean, «Les statues divines du paganisme», dans Ch. Delattre (éd.), Objets sacrés, objets magiques de l'Antiquité au Moyen Âge, Paris, Picard, 2007, p. 53-64.

BOURGEOIS Brigitte \& JOCKEY Philippe, «La dorure des marbres grecs. Nouvelle enquête sur la sculpture hellénistique de Délos », Fournal des Savants, 2005, p. 253-316.

BREMMER Jan, «The Agency of Greek and Roman Statues», Opuscula, 6, 2013, p. 7-21.

BRØNS Cecilie, Gods and Garments. Textiles in Greek Sanctuaries in the 7th1st Centuries BC, Copenhage, University of Copenhagen, 2014.

BRULÉ Pierre, Les sens du poil (grec), Paris, Les Belles Lettres, 2015.

— \& SOURVINOU-INWOOD Christianet, «Athenian Myths and Festivals. Aglauros, Erechtheus, Plynteria, Panathenaia, Dionysia », Kernos, 25, 2012, p. 339-347.

BRUNEAU Philippe, Recherches sur les cultes de Délos à l'époque hellénistique et à l'époque impériale, Paris, De Boccard, 1970.

BURKERT Walter, «From Epiphany to Cult Statue: Early Greek Theos», dans A. B. Lloyd (éd.), What is a God?, Londres, Duckworth, 1997, p. 15-34.

CHANTRAINE Pierre, Dictionnaire étymologique de la langue grecque, Paris, Klincksieck, 1999.

CLASSEN Constance, The Deepest Sense: A Cultural History of Touch, Chicago, The Ilinois University Press, 2012.

CLELAND Liza, The Brauron Clothing Catalogues, Oxford, Hedges, 2005.

CORBETT P. E., «Greek Temples and Greek Worshippers: The Literary and Archaeological Evidence», Bulletin of the Institute of Classical Studies, 17, 1970, p. $149-158$.

DELATTRE Charles, «La statue sur le rivage : récits de pêche miraculeuse», dans Objets sacrés, objets magiques de l'Antiquité au Moyen Âge, Paris, Picard, 2007, p. 65-82.

ESTIENNE Silvia, «Les dévots du Capitole. Le "culte des images" dans la Rome impériale entre rites et superstition», Mélanges de l'École française de Rome, 113, 2001, p. 189-210.

FREEDBERG David, Le pouvoir des images, Paris, Monfort, 1998 (trad. de The Power of Images, 1989).

FRONTISI-DUCROUX Françoise, Dédale. Mythologie de l'artisan en Grèce ancienne, Paris, Maspero, 1975.

GOULD John, «Hiketeia», FHS, 93, 1973, p. 74-103. 
GOUREVITCH Danielle, «Quelques fantasmes érotiques et perversions d'objet dans la littérature gréco-romaine», Mélanges de l'École française de Rome, 94 (2), 1982, p. 823-842.

GRAND-CLEMENT Adeline, La fabrique des couleurs. Histoire du paysage sensible des Grecs anciens, Paris, De Boccard, 2011.

—, «Colori e sensi: percepire la presenza divina», dans G. Pironti \& C. Bonnet (dir.), Gli dèi di Omero. Politeismo e poesia in Grecia antica, Rome, Carocci, 2016, p. 59-84.

GRAVYLENKO Valéria, «Le sens du toucher dans la collection hippocratique», dans G. Puccini (éd.), Le débat des cinq sens de l'Antiquité à nos jours, Bordeaux, Presses universitaires de Bordeaux, 2013, p. 21-32.

HELLER-ROAZEN Daniel, Une archéologie du toucher, Paris, Seuil, 2011 (trad. de The Inner Touch. Archaeology of a Sensation, 2007).

HOLLOWAY Steven W., A šsur is King! Arssur is King!: Religion in the Exercise of Power in the Neo-Assyrian Empire, Leyde, Brill, 2001.

LEKA Euridiki, «L'emploi des huiles, des onguents et des parfums dans l'entretien des statues en Grèce ancienne», dans D. Frère \& L. Hugot (dir.), Les builes parfumées en Méditerranée occidentale et en Gaule, Rennes, Presses universitaires de Rennes, 2012, p. 277-290.

LE GUEN-POLLET Brigitte, La vie religieuse dans le monde grec du $V^{\mathrm{e}}$ au III siècle avant notre ère. Choix de documents épigraphiques traduits et commentés, Toulouse, Presses universitaires du Mirail, 1991.

LHOMMÉ Marie-Karine, «Verrès l'impie : objets sacrés et profanes dans le De signis», Vita latina, 179, 2008, p. 58-66.

LINANT DE BELLEFONDS Pascale, «Rites et activités relatifs aux images de culte», dans Thesaurus Cultus et ritorum antiquorum III.5, Los Angeles, J. Paul Getty Museum, p. 417-507.

LIVINGSTONE Alasdair, «New Dimensions in the Study of Assyrian Religion », dans S. Parpola \& R. M. Whiting (éd.), Assyria 1995: Proceedings of the 10th Anniversary Symposium of the Neo-Assyrian Text Corpus Project Helsinki, Helsinki, 1997, p. 165-177.

LORAUX Nicole, «Le corps vulnérable d'Arès », dans C. Malamoud \& J.-P. Vernant (éd.), Corps des dieux, Paris, Gallimard, 1986, p. 465-492.

MICHELINI Chiara, Storie di statue di Sicilia: tra realtà e immagine, dans C. Ampolo et al., Immagine e immagini della Sicilia e di altre isole del Mediterraneo antico, I, Pise, Scuola Normale Superiore, 2009, p. 231-236. MILES Margaret M., Art as Plunder: The Ancient Origins of Debate about Cultural Property, New York, Cambridge University Press, 2008.

MONTEL Sophie, «Des écrins architecturaux sacralisants? Regards sur la présentation de quelques groupes statuaires du monde grec $\gg$, dans $\mathrm{S}$. Estienne et al. (éd.), Image et religion dans l'Antiquité gréco-romaine, Naples, Centre Jean Bérard, 2008, p. 165-179. 
—, «The Architectural Setting of the Knidian Aphrodite», dans A. C. Smith \& S. Pickup (éd.), Brill's Companion to Aphrodite, Leyde-Boston, Brill, 2010, p. 251-268.

MURGIER Charlotte, «Comment hiérarchiser les sens? Perspectives aristotéliciennes», dans G. Puccini (éd.), Le débat des cinq sens de l'Antiquité à nos jours, Bordeaux, Presses universitaires de Bordeaux, 2013, p. 59-72.

MYLONOPOULOS Joannis, «Divine Images Versus Cult Images. An Endless Story about Theories, Methods and Terminologies», dans J. Mylonopoulos (éd.), Divine Images and Human Imaginations in Ancient Greece and Rome, Leyde, Brill, 2010, p. 1-19.

PARKER Robert, Miasma: Pollution and Purification in Early Greek Religion, Oxford, Clarendon Press, 1990.

PIETTRE Renée, «Images et perception de la présence divine en Grèce ancienne», Mélanges de l'École française de Rome, 113 (1), 2001, p. 211-224.

PIGEAUD Jackie, «La peau comme frontière », Micrologus. La pelle umana, 13, 2005, p. 23-53.

PIRENNE-DELFORGE Vinciane, L'Aphrodite grecque, Athènes-Liège, Centre international d'étude de la religion grecque antique, 1994.

—, «Des marmites pour un méchant petit hermès! ou comment consacrer une statue», dans S. Estienne et al. (éd.), Image et religion dans l'Antiquité gréco-romaine, Naples, 2008a, p. 103-110.

—, Retour à la source Pausanias et la religion grecque, Liège, Centre international d'étude de la religion grecque antique, 2008b.

—, «Greek Priests and 'Cult Statues': In How Far are they Necessary?», dans I. Mylonopoulos (éd.), Divine Images and Human Imaginations in Ancient Greece and Rome, Leyde, Brill, 2010, p. 121-141.

PLATT Verity, Facing the Gods: Epiphany and Representation in Graeco-Roman Art, Literature and Religion. Greek Culture in the Roman World, Cambridge, Cambridge University Press, 2011.

PROST Francis, «L'odeur des dieux en Grèce ancienne : encens, parfums et statues de culte», dans L. Bodiou, D. Frère \& V. Mehl (dir.), Parfums et odeurs dans l'Antiquité, Rennes, Presses universitaires de Rennes, 2008, p. $97-103$.

ROBERT Renaud, «Ambiguïté du collectionnisme de Verrès », dans J. Dubouloz \& S. Pittia (éd.), La Sicile de Cicéron, Lecture des Verrines, Besançon, Presses universitaires de Franche-Comté, 2007, p. 15-34.

ROMANO Irene Bald, «Early Greek Cult Images and Cult Practices», dans R. Hägg \& N. Marinatos (éd.), Early Greek Cult Practice, Stockholm, Aströms, 1988, p. 125-134.

ROMEYER-DHERBEY Gilbert, «Voir et toucher. Le problème de la prééminence d'un sens chez Aristote», Revue de Métaphysique et de Morale, 96 (4), 1991, p. 437-454. 
RUDHARDT Jean, «La perception grecque du territoire sacré», Mélanges de l'École française de Rome, 113, 2001, p. 175-188.

RUTLEDGE Steven H., Ancient Rome as a Museum: Power, Identity, and the Culture of Collecting, Oxford studies in ancient culture and representation, Oxford, Oxford University Press, 2012.

SEAMAN Kristen, «Retrieving the Original Aphrodite of Knidos », Rendiconti della Accademia dei Lincei, 15 (3), 2004, p. 531-594.

SIMON Erika, Festivals of Attica, Madison, University of Wisconsin Press, 2002.

SORABJI Richard, «Aristotle on Demarcating the Five Senses », The Philosophical Review, 80 (1), 1971, p. 55-79.

STEWART Andrew, Art, desire and the Body in Ancient Greece, Cambridge, Cambridge University Press, 1997.

STOICHITA Victor, L'effet Pygmalion. Pour une anthropologie bistorique du simulacre, Genève, Droz, 2008.

THEMELIS Petros, «Artemis Orthia at Messene. The Epigraphical and Archaeological Evidence», dans R. Hägg (éd.), Ancient Greek Cult Practice from the Epigraphical Evidence, Stockholm, Aströms, 1994, p. 101-122.

VERNANT Jean-Pierre, «Corps obscurs, corps éclatants», dans C. Malamoud \& J.-P. Vernant (éd.), Corps des dieux, Paris, Gallimard, 1986, p. 19-58.

WOODFORD Susan, «Heracles Alexikakos Reviewed», American fournal of Archaeology, 80 (3), 1976, p. 291-294. 